Maria Sanz

Sánchez

Figura 1. Climatron (Archivo Roberto Osuna y $M^{a}$ Teresa Valcarce).

Figura 2. Invernaderos del Jardin Botánico de la Universidad de Graz (Roberto Osuna y $M^{a}$ Teresa Valcarce).

Figura 3. Invernadero Santiago Castroviejo del Real Jardin Botánico de Madrid (autora).

\section{La forma del invernadero contem- poráneo y la incidencia de la luz solar}

\author{
Análisis de tres casos: Climatron, Graz y Madrid
}

Palabras clave: invernadero, jardin botánico, luz solar, siglo XX, control climático, carta solar, Climatron, Graz, Santiago Castroviejo. La incidencia de la luz es uno de los aspectos más importantes en el diseño de un
invernadero, debido a su función de recrear en su interior un clima diferente al de
la latitud en la que se encuentra. Sin embargo, aunque pueda parecer que éste es el
factor determinante en la configuración formal de un invernadero, como en todo edi-
ficio, existen otros muchos parámetros a considerar. En este articulo se analizan
tres invernaderos de referencia posteriores a la Segunda Guerra Mundial, y se
observan los aspectos que, en cada caso, han predominado sobre la que sería la
forma ideal en lo que respecta a la incidencia de la luz solar. En el primer inver-
nadero, la cuestión se centrará en la imposición de una forma y sistema estructural
concretos, en el segundo, en la composición dinámica del conjunto, y en el tercero,
en la integración del invernadero en su emplazamiento. Los tres casos tienen en
común el compromiso que presenta su solución entre unos y otros parámetros
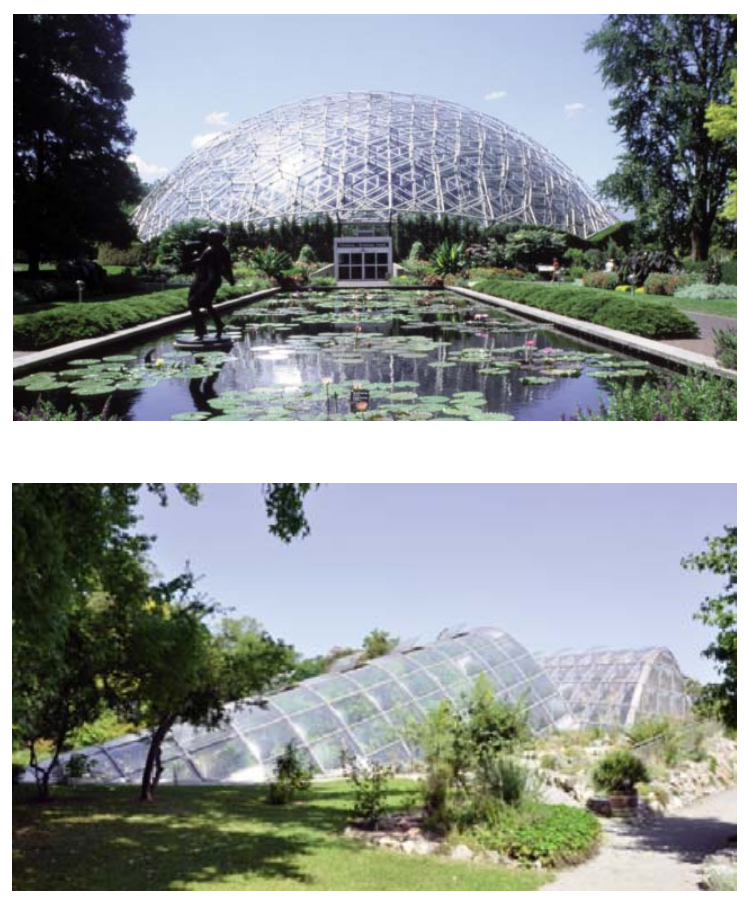

\footnotetext{
Arquitecta por la Escuela Técnica Superior de Arquitectura de Madrid, 2007. Actualmente realizando la tesis doctoral en el Departamento de Composición de la ETSAM
}

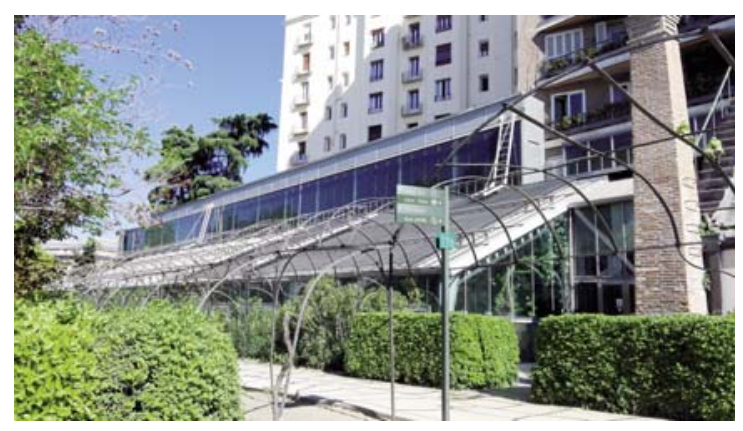

El invernadero de exposición botánica -del siglo XX no ocupa un lugar central en los libros y revistas de arquitectura, ${ }^{1} \mathrm{a}$ diferencia del invernadero del siglo XIX, que está profundamente documentado y publicado. Por ello, el análisis del invernadero del siglo XX constituye un tema de gran interés. En relación a su función, se pueden distinguir dos tipos de invernaderos: el de exposición botánica y el de producción agrícola. Ambos tienen en común el marcado condicionante técnico de optimización del control climático, sin embargo, para el de exposición botánica, la explicación dada en los medios de difusión arquitectónicos, es insuficiente. Debido a esto, con frecuencia, es preciso consultar fuentes de divulgación científica para tener una comprensión global de este tipo de edificios.

Sin lugar a dudas, uno de los principales condicionantes técnicos que influyen en el diseño de un invernadero, es el modo en que incide la luz solar en él. No obstante, aunque pueda parecer que este factor es el determinante en la configuración formal de un invernadero, como en todo edificio, existen otros muchos parámetros que lo definen. En este artículo se analizan tres invernaderos posteriores a la Segunda Guerra Mundial, desde el punto de vista de las relaciones existentes entre su forma y el requerimiento técnico de la optimización de la luz solar, con el fin de observar cuáles han sido los factores que, en cada caso, 
Figura 4. Diferentes secciones para invernaderos propuestas por los predecesores de Loudon. (Hix 1996: 24).

Figura 5. Carta solar de San Luis (ref. web 5).

Figura 6. Carta solar de Graz (ref. web 5).

Figura 7. Carta solar de Madrid (ref. web 5).
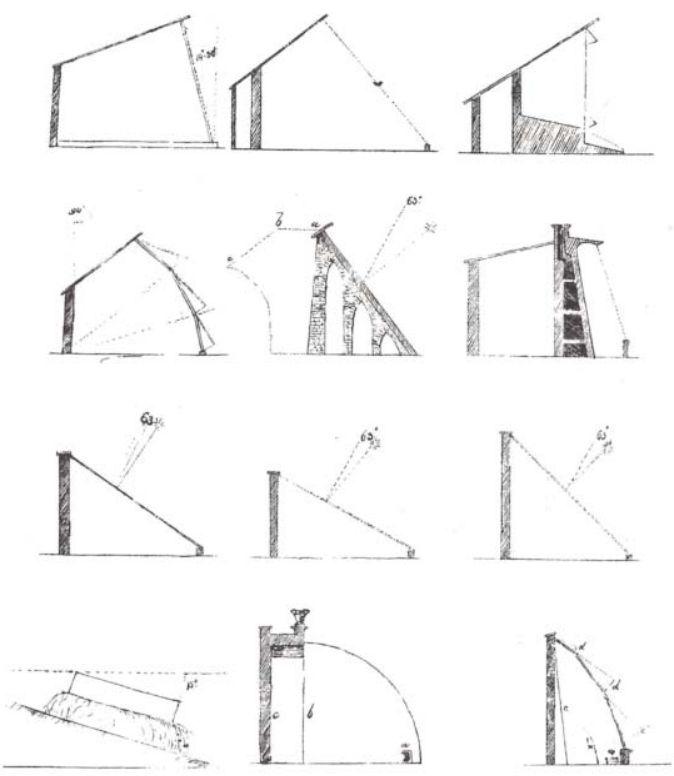

han prevalecido sobre dicho condicionante técnico, dando lugar a un compromiso entre unos y otros aspectos.

Los invernaderos elegidos son: el Climatron del Jardín Botánico de Missouri, los invernaderos del Jardín Botánico de la Universidad de Graz y el invernadero Santiago Castroviejo del Real Jardín Botánico de Madrid (figuras 1, 2 y 3).

De entre todos los invernaderos de exposición construidos después de la Segunda Guerra Mundial, se han elegido estos ejemplos por considerarlos invernaderos de referencia por su solución, y además porque cada uno de ellos representa un tipo diferente de configuración formal dentro de los tipos de invernaderos contemporáneos: el Climatron es una gran cúpula geodésica, los invernaderos de Graz, un conjunto de cilindros parabólicos, y el invernadero de Madrid está compuesto por una cubierta inclinada acristalada a lo largo de un muro de contención. El hecho de que sean posteriores a la Segunda Guerra Mundial es debido al cambio radical que se puede observar en el diseño de los invernaderos construidos con posterioridad a dicha guerra, a los que en este artículo denominamos "contemporáneos". La Segunda Guerra Mundial es el elemento de ruptura con el estilo neo-victoriano que caracterizó los invernaderos construidos con anterioridad a ella, considerados, por tanto, herederos del siglo XIX, a pesar de pertenecer al siglo $\mathrm{XX}^{2}$

Las primeras referencias significativas acerca de la forma del invernadero en relación al modo en que incide la luz solar, se remontan a los tratados de principios del siglo XIX, siendo John Claudius Loudon el autor de referencia de dicho siglo. ${ }^{3}$ En su tratado Remarks on the Construction of Hothouses, incluye el estado de la cuestión hasta ese momento, y recoge e ilustra, con secciones de invernaderos, las propuestas de sus predecesores acerca de la envolvente de vidrio en relación a la captación de luz (figura 4).

Su Encyclopedia of Gardening, tiene como idea clave que "la introducción y organización de la luz es el punto más importante a tener en cuenta en la construcción de invernaderos". (Loudon 1822: 354). En esta obra, Loudon por primera vez publica un catálogo de formas curvilíneas nuevas para el edificio de invernadero, a partir de la observación directa de prototipos. (Loudon 1822: 357 - 358).

Estas propuestas se basan en el principio del ángulo de incidencia del sol, que "deriva de la conocida ley óptica, según la cual, la influencia del sol en cualquier superficie (...) es directamente proporcional a su per-
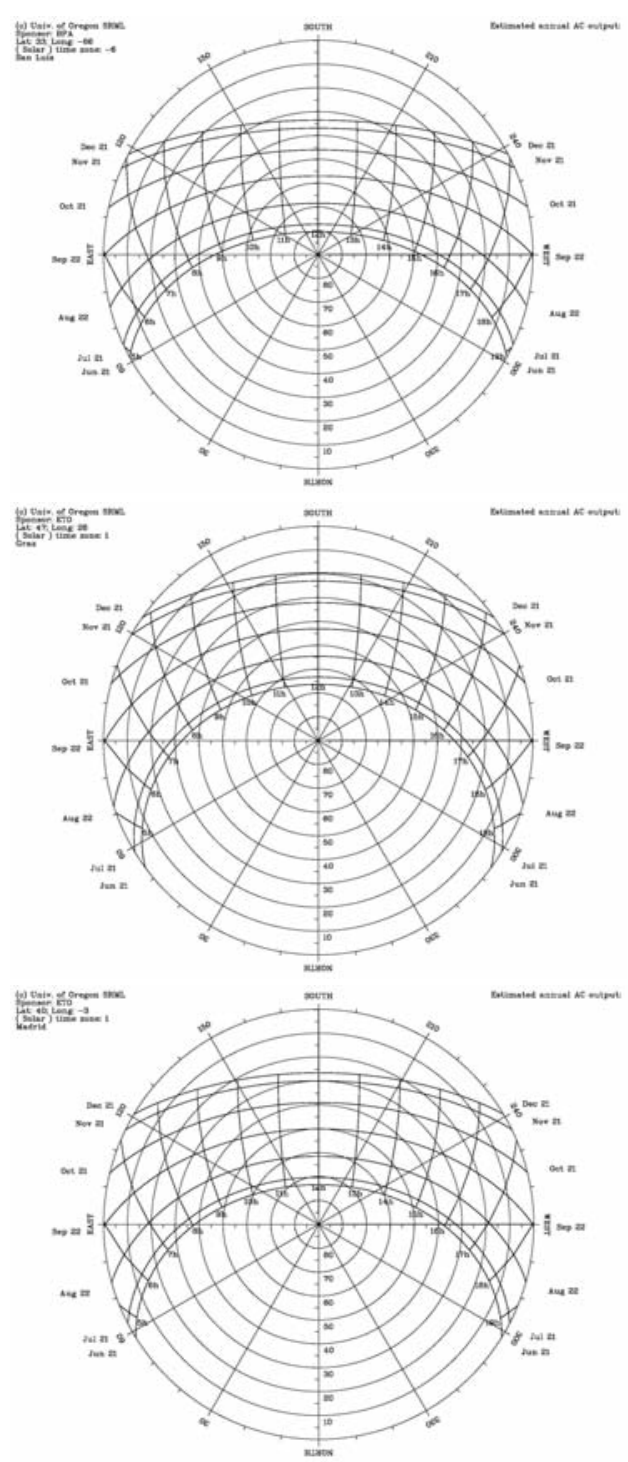
Figura 8. Planta general del Jardin Botánico de Misuri 1974-1977 (http://www.wikiwand.com/en/Missouri_Botanical_Garden). Simbolo de norte por la autora.

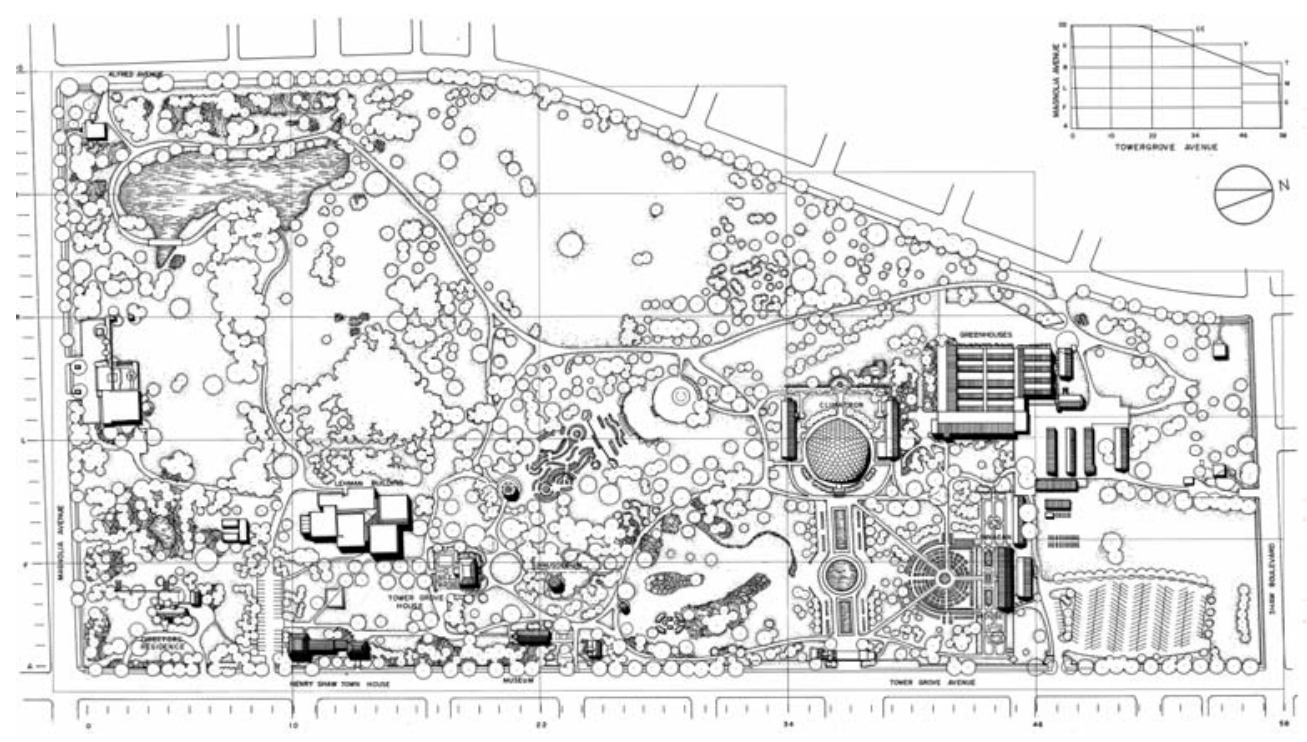

pendicularidad a dicha superficie". (Loudon 1822: 354). Considerando dicha ley óptica, o Ley de Snell, se generó una serie de propuestas de inclinación de la superficie de vidrio, en función de la época del año en que se requiriera la máxima incidencia de los rayos solares. Según Loudon "el ángulo comprendido entre la pared trasera del invernadero y el plano inclinado de cubierta, siempre deberá coincidir con la altitud del sol, cuando sus rayos caen perpendiculares a ese plano". (Loudon 1822: 355). Para ello, publicó la fórmula a la que previamente había llegado el jardinero T. Wilkinson: "habiendo determinado en qué estación queremos tener el máximo efecto del sol, debemos construir nuestro invernadero de acuerdo con la siguiente regla: ángulo comprendido entre la pared opaca y la cubierta = colatitud del lugar + /- declinación del sol en el día en que queremos que los rayos caigan perpendiculares. Desde el equinoccio de primavera hasta el de otoño, la declinación se suma, y a la inversa". (Wilkinson 1812, citado en Loudon 1817: 10). ${ }^{4}$

El principio del ángulo de incidencia del sol tiene su aplicación en el análisis de los tres invernaderos posteriores a la Segunda Guerra Mundial elegidos, realizando para cada uno un diagrama solar en su sección norte-sur, de la siguiente manera: en las cartas solares estereográficas ${ }^{5}$ de cada invernadero se ha seleccionado la altura solar máxima a las 12:00, hora solar, en el solsticio de verano y en el solsticio de invierno (figuras 5, 6 y 7), y se han representado estos dos datos en su sección norte-sur. 6

Este diagrama permite observar con qué ángulo inciden los rayos solares en la envolvente del edificio en estos dos momentos del año y reflexionar sobre las relaciones geométricas existentes entre la forma del invernadero y el recorrido del sol, detectando qué factores han alterado la forma que sería óptima en relación a la optimización de la luz solar. Por ejemplo, si la envolvente es perpendicular a los rayos solares en el solsticio de verano, captará la máxima luz con la mínima energía reflejada en esa estación, y si es oblicua a los rayos solares, su función será reflejar la máxima cantidad de luz posible, protegiendo del sobrecalentamiento.

El primero de los invernaderos analizados es el Climatron del Jardín Botánico de Misuri, inaugurado en 1961. Este invernadero es una gran cúpula geodésica de aluminio de 53,34 $\mathrm{m}$ de diámetro y 21,33 m de altura, diseñada con una patente de Buckminster Fuller (figura 1). Representa a aquellos invernaderos contemporáneos resueltos mediante una cúpula de enormes dimensiones. El Jardín Botánico de Misuri fue fundado en San Luis en 1859 y es uno de los jardines botánicos de referencia en Estados Unidos. El emplazamiento del Climatron está directamente condicionado por la organización del jardín histórico al que pertenece, ya que se sitúa exactamente en el lugar en el que estaba la Palm House a la que sustituye, un antiguo edificio compuesto según "el tradicional esquema victoriano de edificio en forma de E con una cúpula en el centro". (Cunningham 2000: Introducción vii) (figura 8).

Si se analiza en detalle la sección del Climatron, se observa que la cúpula es una porción de esfera cortada por el plano del suelo, a una altura más elevada que donde se encuentra el centro de dicha esfera (figura 9). Este gesto hace que la altura de la cúpula se reduzca, y por tanto que las plantas se sitúen más cerca de la superfi- 
Figura 9. Esquema geométrico de la sección del Climatron (autora).

Figura 10. Semicúpula propuesta por G. Mackenzie (Hix 1996: 32).

Figura 11. Diagrama solar norte-sur del Climatron (autora).

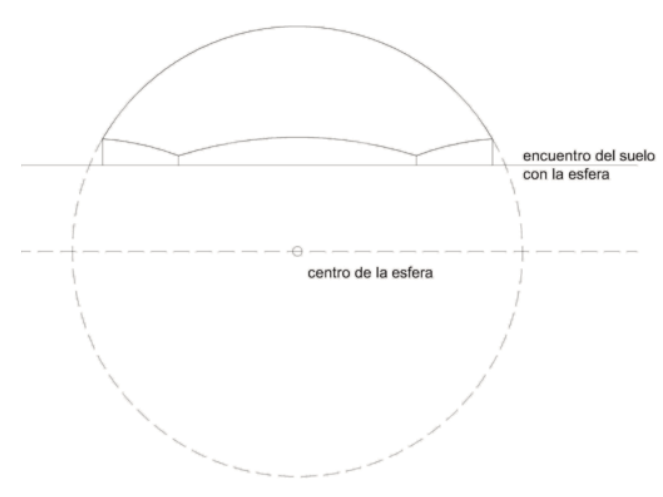

lugar de una semicúpula, para este invernadero se optó por una cúpula de Fuller completa, lo cual es cuestionable en términos de control climático, ya que, como se ha comprobado, esta forma da lugar a una amplia zona del invernadero hacia el norte en la que no se optimiza la incidencia de la luz solar.

Conviene preguntarse cuáles fueron los motivos que llevaron a la elección de esta forma, por encima de las consideraciones técnicas relativas exclusivamente a la incidencia de luz solar. La respuesta se encuentra en los dos principales requisitos de partida del proyecto. En primer lugar, el nuevo invernadero debería ser un nuevo símbolo con carácter monumental para el jardín. El Doctor Wert, director de la institución, afirmaba en la Journal of the American Institute of Architects: "necesitábamos sustituir el viejo invernadero por una estructura monumental que fuera la característica y la atracción principal del jardin". ("The Climatron, Missouri" 1961: 27). En segundo lugar, debía tener unas caracteristicas espaciales particulares, ya que tenía que "permitir el crecimiento de las plantas de diferentes ecosistemas bajo condiciones prácticamente naturales y ser un espacio natural y atractivo". Por ello se requería de un "enorme espacio interior sin sombras arrojadas, con gran intensidad lumínica y control ambiental para recrear un ambiente de clima tropical". (“The Climatron, Missouri” 1961: 27).

La cúpula geodésica, no sólo respondía a criterios de optimización de la luz solar, sino que además garantizaba la monumentalizad y la espacialidad requeridas. Los propios arquitectos reconocían que "la elección de una cúpula geodésica (bajo los principios de Buckminster Fuller) aportó una 'estructura monumental' — de aproximadamente el diámetro del Panteón- permitiendo soluciones para todos los requerimientos". ("The Climatron, Missouri" 1961: 30). Además, la cúpula geodésica
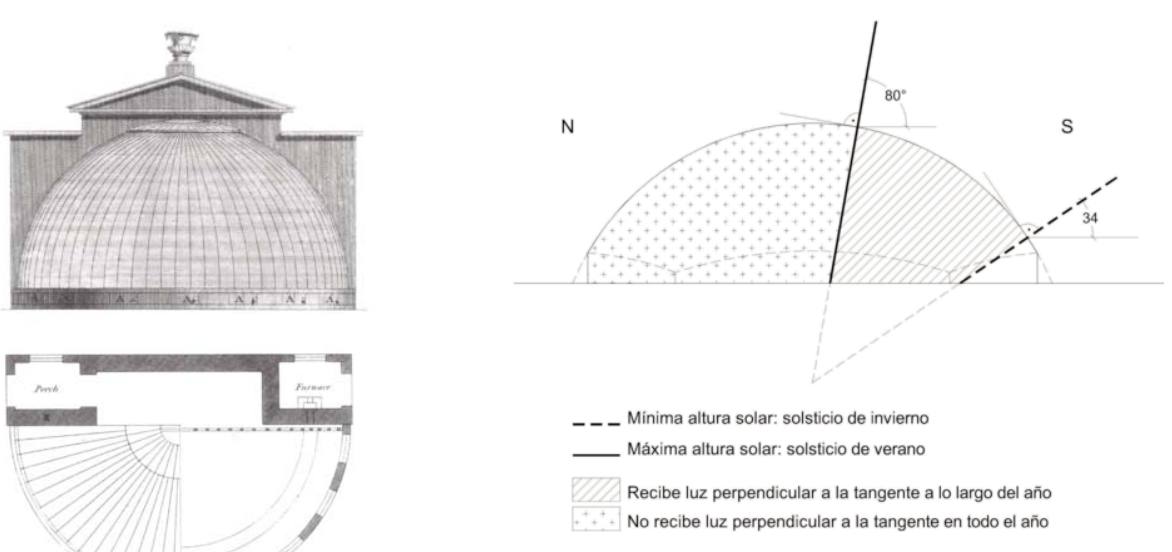
Figura 12. Vista interior del Climatron (Roberto Osuna y $M^{a}$ Teresa Valcarce).
Figura 13. Planta del Climatron. Los doce climas tropicales se distribuyen en sectores de circunferencia y los sistemas de aire acondicionado en los ejes sur-este y oesteeste (dibujo de la autora basado en "Tropics on the" 1961: 114).

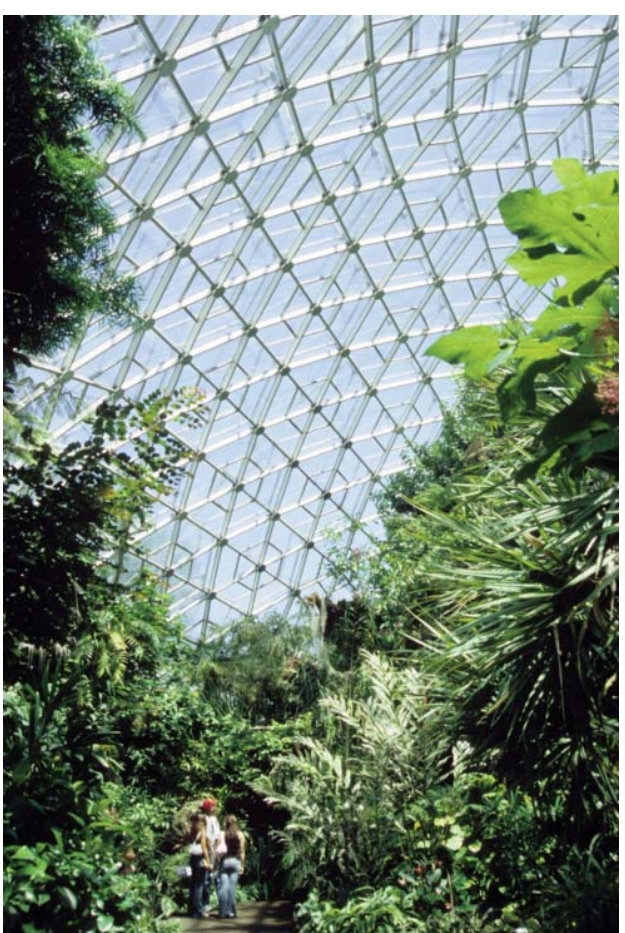

aportó las características espaciales deseadas, ya que, al no necesitar soportes intermedios, dio lugar a un interior totalmente diáfano con "una mínima sensación de envolvente desde el interior". ("The Climatron, Missouri” 1961: 27) (figura 12).

Dentro del único espacio interior del Climatron, se recrean cuatro áreas tropicales, dispuestas en sectores de circunferencia, cuyos matices dan lugar a doce climas "con diferentes demandas de temperatura y humedad, variando durante el día y la noche" ("Science in Overseas" 1961: 362). La respuesta acerca de cómo se obtienen

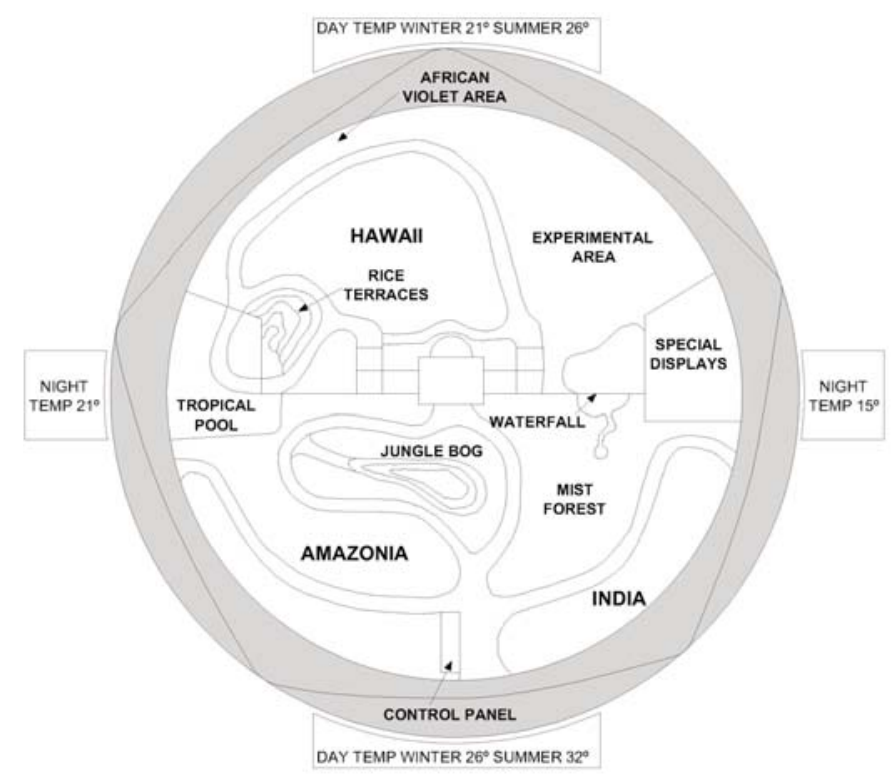

estas variaciones climáticas sin compartimentar el espacio, está en el sistema de acondicionamiento empleado, compuesto por "dos sistemas independientes de aire acondicionado, uno para calentar — para simular la ganancia de calor solar en invierno-, y otro para enfriar (...) en verano" ("Tropics on the" 1961: 218). El aire es tratado desde todo el perímetro de la cúpula, ya que "el sistema de calefacción mueve aire desde el lado sur del Climatron hacia el norte; el de enfriamiento, lo mueve desde el oeste hacia el este" ("Tropics on the" 1961: 218). ${ }^{8}$ De modo que, debido al recorrido del aire, en el interior de la cúpula se crea un gradiente de temperatura y humedad para el acondicionamiento térmico de cada sector de circunferencia (figura 13). Así, el Climatron se convirtió en "el primer invernadero con aire acondicionado" (Elwall 2001: 130). Debido a que estos dos sistemas funcionan en el perímetro, según los ejes sur-norte y oesteeste, es necesario que el volumen del edificio sea simétrico respecto de su centro, por lo que sería contradictorio situar un muro opaco hacia el norte, tal y como se ha propuesto antes a la luz del diagrama solar. Es decir, la forma del Climatron está estrechamente relacionada con su sistema de acondicionamiento.

En el aspecto constructivo, "la estructura geodésica de perfiles de aluminio descansa en cinco pilares de hormigón, separados equidistantemente a lo largo de la base circular, entre pilar y pilar se forma un arco con el que se encuentra el entramado metálico". (Woods, Swartz 1988: 182). La envolvente está compuesta por piezas triangulares de Plexiglas, un novedoso material cuya ligereza y flexibilidad lo hacían muy apropiado para este tipo de estructuras. "Este sistema en realidad duró 28 años, aunque hubo fugas desde el principio, sin duda causadas por el movimiento de la estructura flexible. A lo largo de los años la piel se desgastó y surgieron pequeñas fracturas internas, dando al inicialmente luminoso Climatron una apariencia nebulosa, y reduciendo su transmisión luminosa en un 50\%." (Freeman 1989: 88). Para devolver a la cúpula su transparencia, en 1989 se retiraron las piezas de Plexiglas y se construyó una segunda cúpula interior con un revestimiento de vidrio bajo emisivo (figura 14).

La elección de la cúpula de Fuller, no sólo modificó la forma que habría sido ideal para el invernadero en relación a la optimización de la luz solar, sino que además se convirtió en el mayor símbolo del Jardín Botánico, tal y como se buscaba desde el 


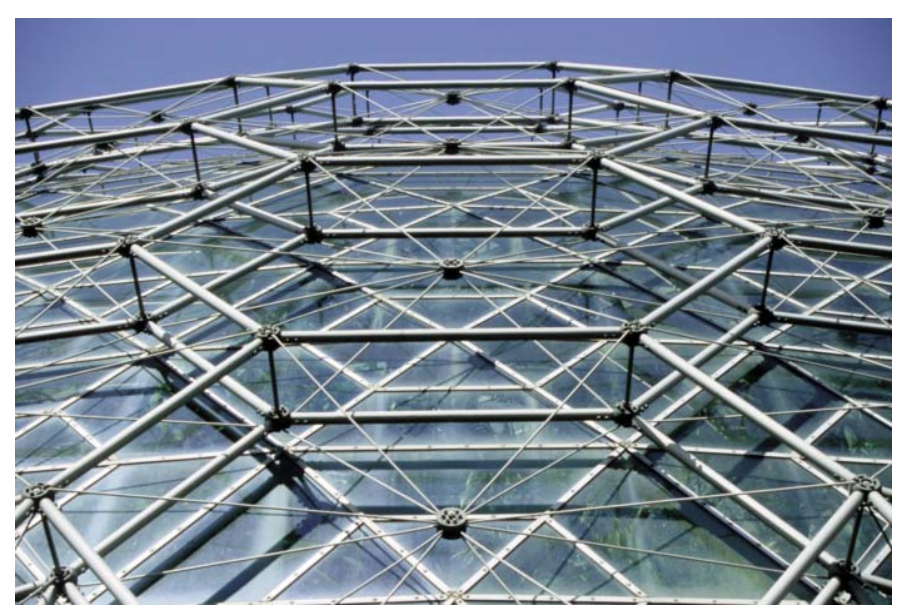

Figura 14. Vista exterior de las dos cúpulas (Roberto Osuna y $M^{a}$ Teresa Valcarce).

Figura 15. Planta general del conjunto. (Baldassini 1997: 22) (simbolo de norte por la autora). principio. Los autores de este invernadero, Murphy \& Mackey, obtuvieron el Reynolds Award en 1961, lo que tuvo gran difusión en las publicaciones especializadas, que se hicieron eco de la descripción dada por el jurado. Este es el caso de la Journal of the American Institute of Architects en su articulo "The Climatron", de Architectural Forum, en su artículo "Climatron wins Reynods Award", o de la revista Architectural Record en su artículo "Murphy \& Mackey win 1961 Reynold Award", donde afirmaban que "esta aplicación de los principios de Buckminster Fuller en una cúpula geodésica está ejecutada con sensibilidad y es sorprendentemente apropiada 30, "Murphy \& Mackey win 1961 Reynold Award" 1961: 12, "Climatron wins Reynols Award" 1961: 5). Así mismo, la Journal of the American Institute of Architects, en el mencionado artículo, lo describía como "una bella y refinada versión de una de las más antiguas formas arquitectónicas" ("The Climatron" 1961: 30). Además, la para su propósito" ("The Climatron" 1961: sensación espacial conseguida ha sido muy positivamente valorada por dichos medios: "El Climatron es un punto y final, la tecnología del artefacto / arquitectonica, es tan potente, que su expresión formal / espacial es como una telaraña, modesta, minimalista. La naturaleza ha ganado" (Riley 1997: 45).

El funcionamiento de los sistemas de control climático se describe detalladamente en el citado artículo de la Journal of the American Institute of Architects ("The Climatron" 1961) donde se afirma que "no sólo la organización y el diseño, sino también los aspectos estructurales y mecánicos del problema son de gran interés". Más allá de los medios de difusión arquitectónicos, la explicación sobre el sistema de control climático fue extensamente publicada en revistas de divulgación científica. ${ }^{9}$

Finalmente, cabe destacar que el Climatron es un edificio de referencia dentro de la arquitectura del siglo XX, no sólo porque responde a los condicionantes técnicos propios de un invernadero, sino también a los requisitos particulares del proyecto. $\mathrm{Su}$ solución formal alcanza un alto grado de compromiso entre la búsqueda de la monumentalidad y la espacialidad deseadas, y la adecuada incidencia de la luz solar, para disfrutar de doce climas tropicales diferentes en un único espacio.

El segundo caso analizado es el conjunto de invernaderos del Jardín Botánico de la Universidad de Graz, del arquitecto Volker Giencke. Proyectado en 1983 y construido en 1992, se encuentra ocupando la esquina noreste de una de las manzanas ortogonales del jardín. El conjunto lo componen cuatro volúmenes, uno de ellos con función de vivero, y tres con función de invernadero, cada uno con condiciones climáticas diferentes: clima templado $\mathrm{y}$ seco, clima tropical y clima frío) (figura 15).

Al igual que el Climatron representa a los invernaderos contemporáneos con forma de cúpula, éstos representan aquellos invernaderos contemporáneos con forma longitudinal, generada por la sucesión de una única sección transversal, la cual es una forma geométrica pura. En este caso, la sección es una parábola (figura 16), de modo que "el edificio consta de tres cilindros parabólicos que penetran diagonalmente entre sî". ("Volker Giencke: glasshouses" 1996: 62). 


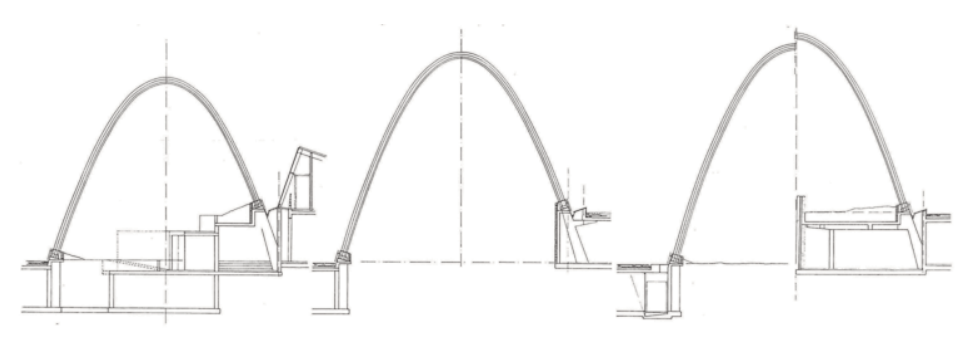

Figura 16. Secciones transversales del invernadero de clima templado (Baldassini 1997: 23).

Figura 17. Diagrama solar norte-sur del invernadero de clima templado (autora).

Figura 18. Foto aérea de la parcela (https://www.google.es/maps).

El invernadero de mayor tamaño "contiene el departamento de clima templado en su extremo norte, la casa de las suculentas al sur, y el nexo de circulación de todo el complejo que ocupa su centro". (BlundellJones 1995: 50/10). Según el diagrama solar norte-sur (figura 17), se observa que los rayos solares, en ningún momento del año inciden de manera perpendicular a la tangente de la envolvente. Esto es debido a que, a diferencia del Climatron, simétrico respecto de su centro, este invernadero tiene una disposición lineal y, tal y como afirma Blundell-Jones, "como edificio lineal su mejor disposición es a lo largo de un

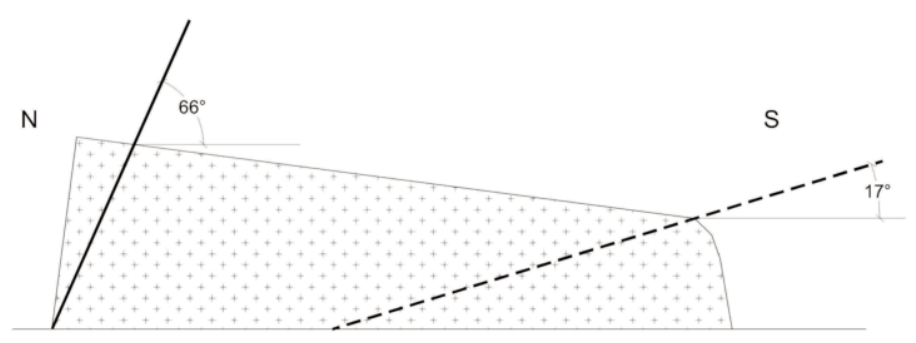

- - - Mínima altura solar: solsticio de invierno Máxima altura solar: solsticio de verano

Recibe luz perpendicular a la tangente a lo largo del año No recibe luz perpendicular a la tangente en todo el año

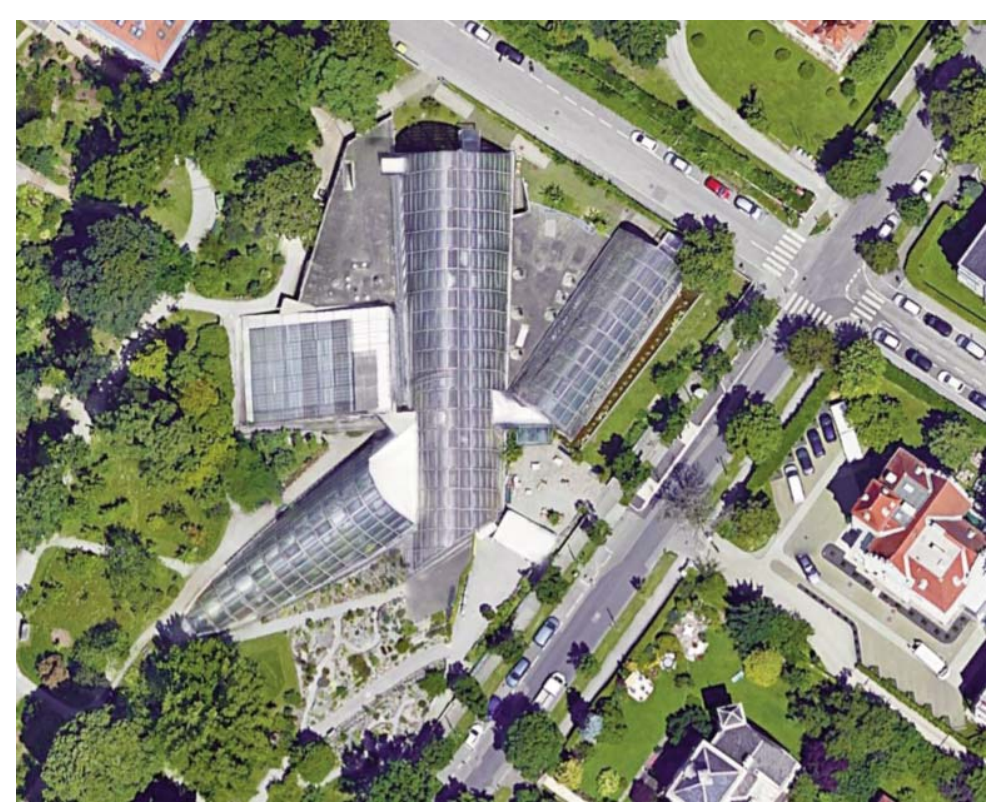

eje norte-sur para recibir el sol a lo largo de todo su recorrido diario". (BlundleJones 1995: 48/10). Esto se ha conseguido girando el edificio $35^{\circ}$ con respecto a la cuadrícula de la calle (figura 18), con el fin de disponer la sección transversal parabólica en dirección este-oeste, ocupando el edificio la mayor diagonal de la parcela. Además, "inclinando el volumen a lo largo de su eje hacia el sur, aumenta la transmisión luminosa a medio día además de dramatizar la relación del edificio con el terreno". (Blundle-Jones 1995: 50/10). Por lo tanto, queda demostrado que la forma y orientación de este invernadero son adecuadas en relación a la optimización de la luz solar, habiéndose impuesto este parámetro por encima de los condicionantes de carácter urbano.

Por el contrario, los otros dos invernaderos conservan la misma sección transversal parabólica, pero no tienen orientación norte-sur, 10 debido a que "en ambos casos se ha abandonado la orientación ideal en favor de la relación con el lugar". (BlundleJones 1995: 50/10) (figura 19).

Cabe preguntarse entonces los motivos que han llevado a emplear la misma sección para todos ellos, en lugar de adaptar su forma a las necesidades climáticas de cada uno, y a su emplazamiento. La respuesta se encuentra en que, del mismo modo que la forma del Climatron estaba previamente definida mediante el empleo de una cúpula de Fuller, para los invernaderos de Graz, el criterio de partida era solucionar todo el conjunto con una sola sección transversal parabólica a lo largo de un eje longitudinal. Como razones para esta decisión, se han publicado argumentos de indole económica: "por motivos de economía ingenieril, se usa el mismo arco como elemento común en los tres invernaderos, con la excepción de una fragmentación a lo largo de la calle. La diferencia entre los invernaderos de clima tropical, frío y templado se encuentra en las secciones de los cilindros parabólicos resultantes y su correspondiente inclinación y posición en el terreno". (Connah 2001: 155). Pero sobre todo, el empeño por resolver todo el conjunto con una misma sección transversal parabólica, responde a una intención inicial del proyecto de dotar a la composición de un gran dinamismo y unas cualidades espaciales particulares. En efecto, "una situación espacial absolutamente nueva y fascinante (...) surge como consecuencia de la penetración concéntrica o el contacto tangencial de los volúmenes" (Connah 2001: 155) (figura 20). 


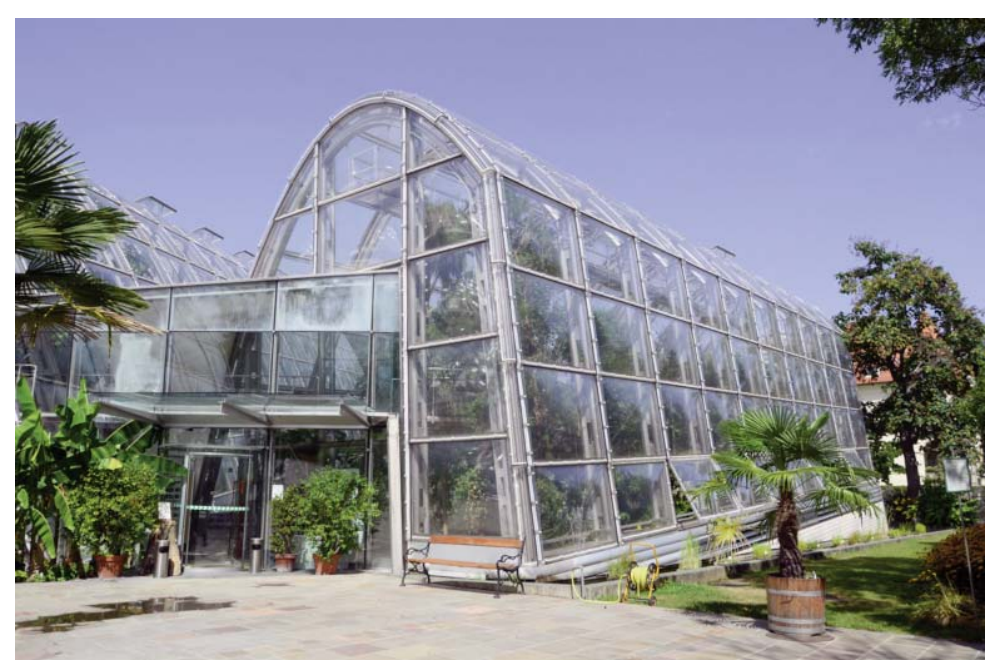

Figura 19. Invernadero de clima frío. Su sección parabólica está modificada para crear un cierre frente a la calle (Roberto Osuna y $M^{a}$ Teresa Valcarce).

Figura 20. Los volúmenes penetran $y$ contactan entre si (Roberto Osuna y $M^{a}$ Teresa Valcarce).

Figura 21. Vista interior del invernadero (Roberto Osuna y $M^{a}$ Teresa Valcarce).
Dada la importancia de esta sección parabólica, merece la pena analizar cuáles son sus cualidades para responder adecuadamente a los requisitos propios de un invernadero. Por un lado, ofrece conocidas ventajas estructurales, ya que "una parábola es la forma ideal para hacer frente a la una distribución homogénea de las cargas y extremadamente similar a la curva catenaria, que en realidad es el mejor modo posible de dispersar el peso". (Baldassini 1997: 23). También responde satisfactoriamente a las necesidades de control climático específicas del edificio, debido a las propiedades geométricas propias de su factor de forma. El propio Blundell-Jones destaca a este respecto que "la sección parabólica, alta en su centro, permite que los árboles crezcan sin generar un excesivo volumen o superficie de envolvente, situando las fachadas laterales perpendiculares al sol rasante del invierno por la mañana y por la tarde" (Blundell-Jones 1995: 48), además, favorece el adecuado crecimiento de las diferentes especies, pues "limita el ancho de la luz haciendo el edificio suficientemente estrecho para permitir a todas las plantas el correcto acceso a la luz solar" (Blundell-Jones 1995: 48). Pero más allá de todas estas cualidades de carácter técnico, la principal aportación de la sección parabólica a estos invernaderos son sus propiedades espaciales. Sobre esto, Niccolò Baldassini afirmaba en la revista L'Arca que "Giencke ha sustituido la típica bóveda de cañón y las formas geométricas derivadas del círculo por una cubierta con forma parabólica, que es más práctica para árboles de gran porte, y significativamente mejor para aportar esa sensación tridimensional inherente a la posición e inclinación de los cuatro pabellones". (Baldassini 1997: 23) (figura 21).

Además, la sección parabólica no sólo determina en gran medida la forma y el espacio de los invernaderos, sino también su construcción y su sistema de acondicionamiento térmico. Como consecuencia de la optimización de la estructura que aporta esta sección, la construcción se caracteriza por el empleo de sistemas y materiales ligeros con secciones reducidas al mínimo para obtener la máxima transmisión luminosa. ${ }^{11}$ La estructura consiste en una sucesión de arcos de sección parabólica unidos por perfiles horizontales, siendo el material estructural el aluminio, debido a "su ligereza y por su resistencia a la corrosión, aunque se han añadido cables tensores contra las cargas de viento". (Jeska 2008: 152). Finalmente, "la envolvente la constituyen unos elementos pásticos de doble capa que revisten la estructura portante como una piel". ("Volker Giencke: glasshouses" 1996: 62). En relación a este revestimiento, en el libro Transparent plastics, nuevamente se alude
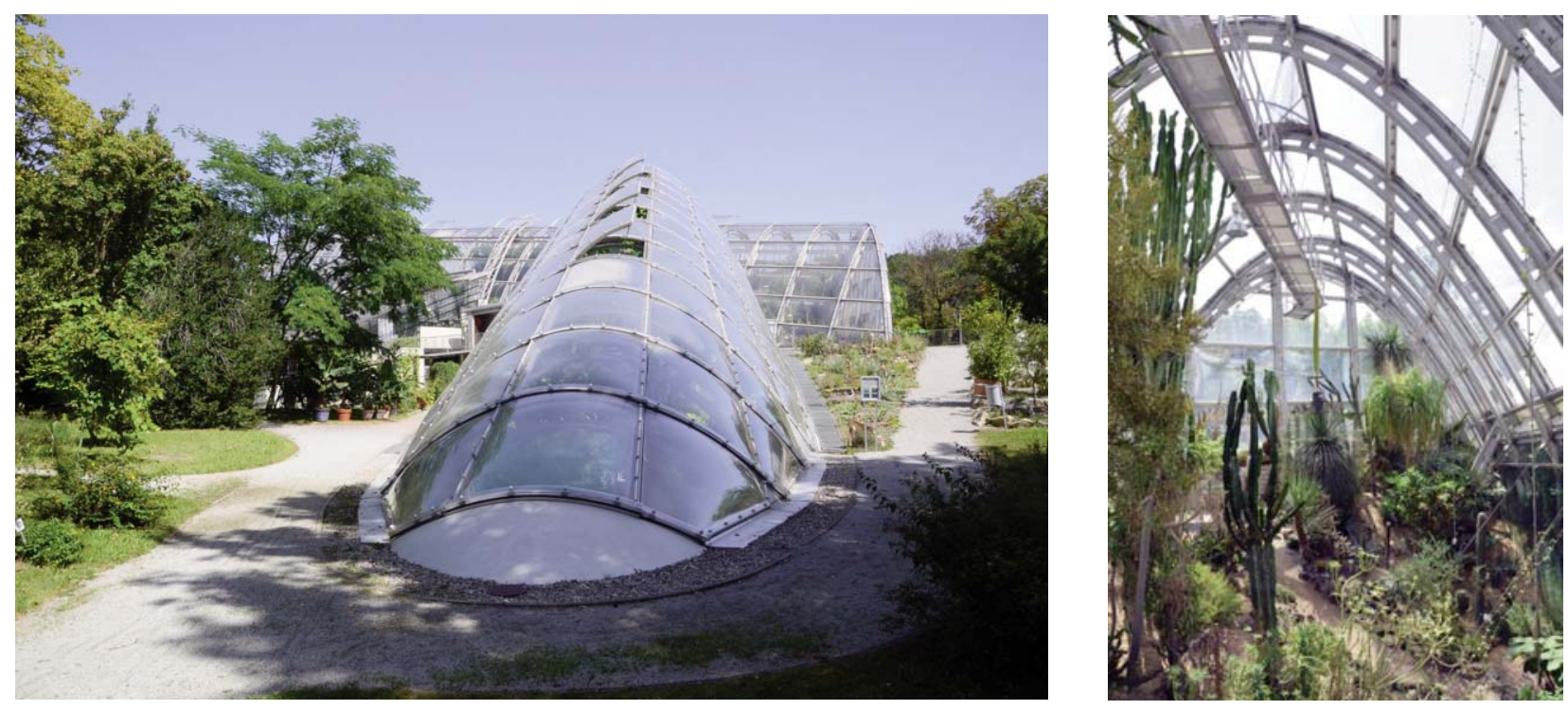


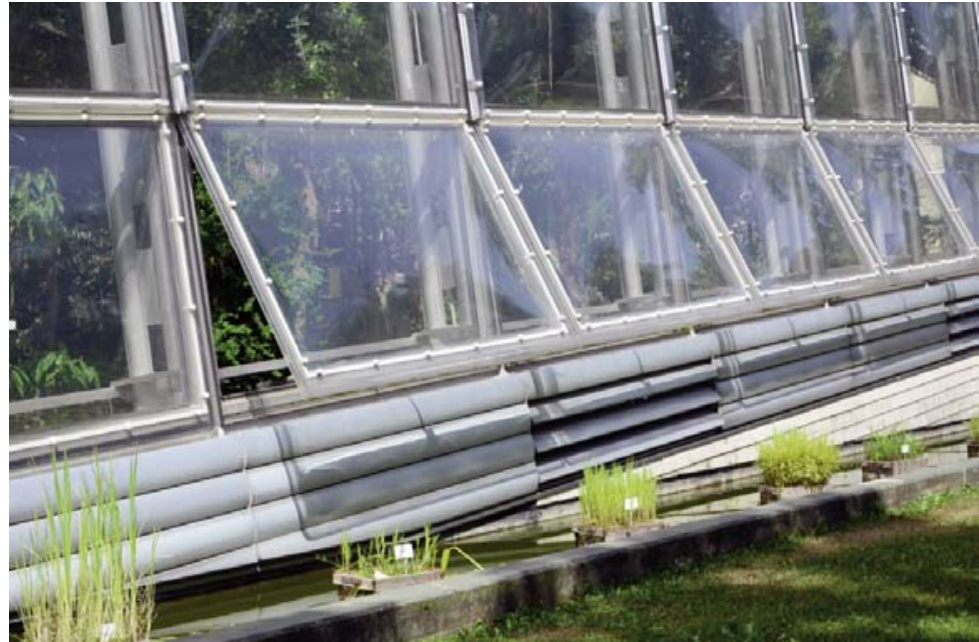

Figura 22. Aberturas inferiores para la entrada de aire (Roberto Osuna y $M^{a}$ Teresa Valcarce).

Figura 23. Vista general del invernadero en el Jardin Botánico de Madrid (autora). a la economía de formas mencionada, ya que "a pesar de la geometría curva de los edificios, los elementos de fachada se pudieron estandarizar porque los tres invernaderos siguen la misma curva parabólica". (Jeska 2008: 152). Por otro lado, el sistema de calefacción está también fuertemente condicionado por la forma del edificio: "como las secciones estructurales son huecas, se bombea agua caliente por ellos, de modo que actúan como si fueran unos radiadores gigantes. El sistema de enfriamiento se hace, en primer lugar por ventilación, introduciendo aire en el nivel inferior y expulsándolo por las aberturas en el vértice. Y en segundo lugar, en particular en el invernadero de clima frío, pulverizando periódicamente una fina niebla de gotas de agua desde tuberias situadas en la parte superior". (Blundell-Jones 1995: 50/10) (figura 22).

Estos invernaderos han tenido una gran difusión en los medios especializados, siendo la eficiencia de los sistemas de control climático, uno de los aspectos más valorados. En la revista Arca, en enero de 1997, se afirmaba que "así como el espacio cumple con los factores constructivos, la estructura del edificio está orientada a fines bioclimáticos: el agua caliente centralizada que atraviesa la sección de los arcos convierte los elementos principales de la construcción en lo que podría describirse como un auténtico radiador urbano que controla la temperatura de los cultivos" (Baldassini 1997: 23). En este sentido, Blundell-Jones sostenía en Architectural Review, de octubre de 1995, que "el uso de un tipo de vidrios especiales, en capas dobles o incluso triples, junto con estructuras ligeras y mecanismos eléctricos de apertura, teóricamente puede permitir un mayor grado de control climático que un invernadero tradicional". (BlundellJones 1995: 50/10). Así mismo, también se han destacado las cualidades espaciales y el dinamismo que caracterizan la composición del conjunto: "volviendo a la cuestión de la sensación espacial, se ha abandonado el estatismo estructural de los invernaderos del siglo XIX (...) a favor de un mayor dinamismo". (Baldassini 1997: 23).

Así, los invernaderos de Graz son un referente dentro de la arquitectura del siglo $X X$. En su solución formal se ha considerado la adecuada incidencia de la luz solar, pero además, se ha aportado una dinámica configuración espacial. Además, el empeño por resolver todos los invernaderos con una única sección parabólica, ha dado lugar a una gran coherencia y unidad entre sus aspectos formales y técnicos.

Por último, el tercer caso analizado es el Invernadero Santiago Castroviejo, del arquitecto Antonio Fernández Alba, construido entre 1991 y 1993 en el Real Jardín Botánico de Madrid (figura 23). A semejan-

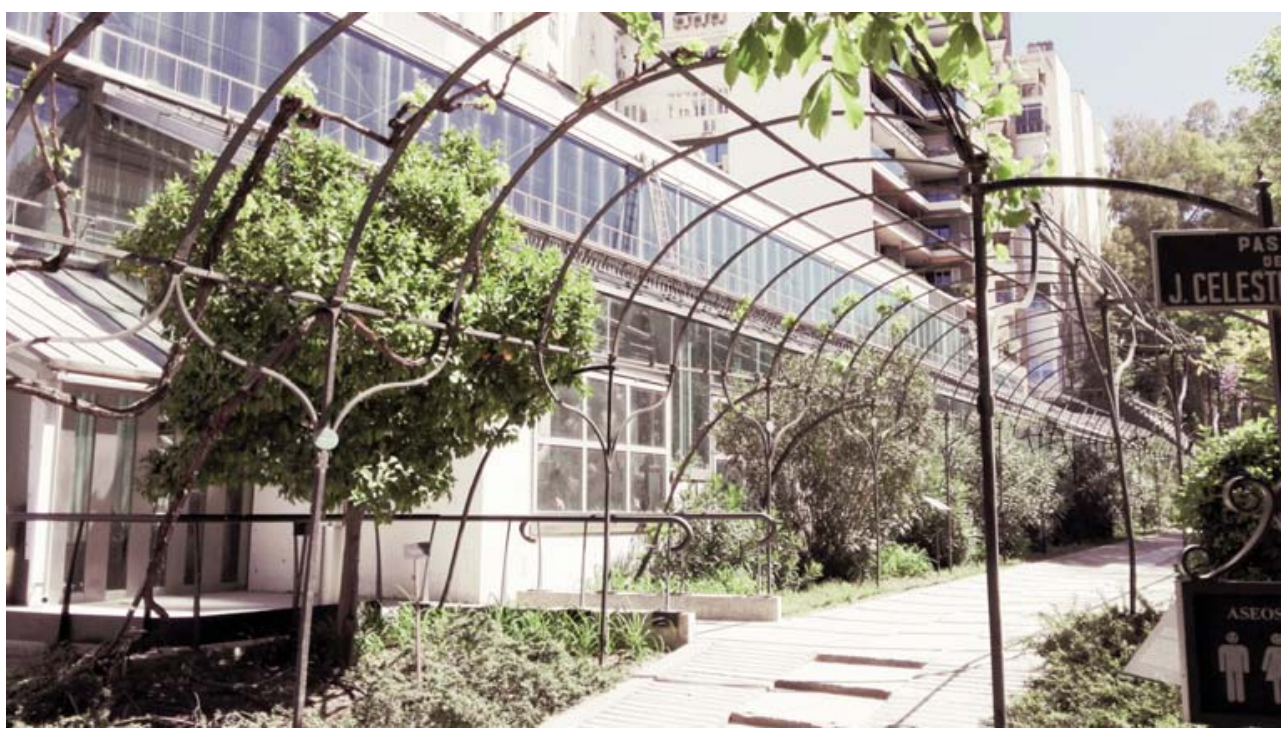



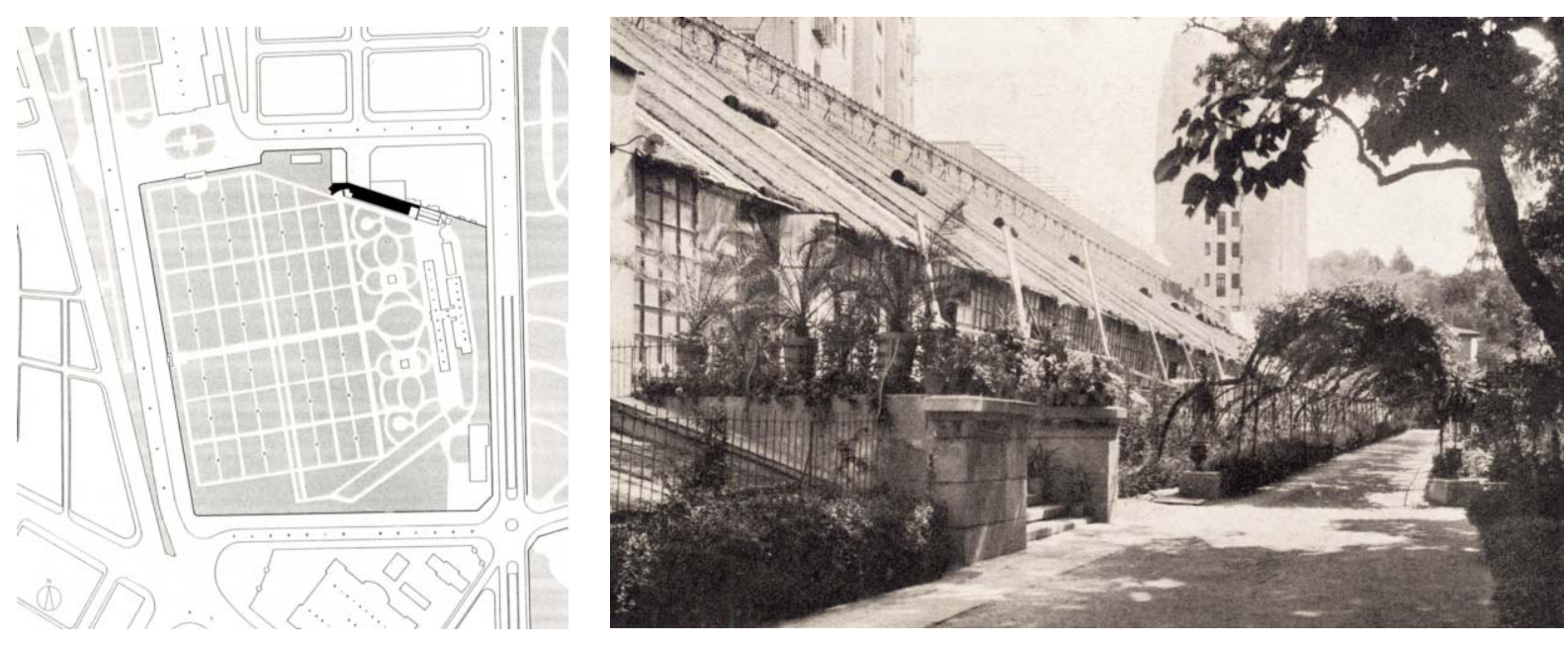

Figura 24. Plano de situación del Jardin (AFA Arquitectos, S.L.P.) Simbolo de norte por la autora.

Figura 25. Antigua estufa del mediodía. (Archivo del Real Jardin Botánico-CSIC, División I, Colección Fotográfica).

Figura 26. Axonometría del Invernadero Santiago Castroviejo y el Invernadero de Graells situado en su limite sureste (AFA Arquitectos, SLP). za de los dos invernaderos anteriores, éste representa a los invernaderos contemporáneos con forma longitudinal, generada a partir de la sucesión de una única sección transversal que contiene un muro de contención. El primer referente de este tipo de sección lo encontramos en el invernadero holandés del siglo XVIII: "los holandeses habian desarrollado espacios con cubiertas inclinadas de vidrio (...). La pared trasera y el suelo eran de mampostería, que absorbía los rayos solares y retenía el calor por la noche. (...) La fachada sur era totalmente de vidrio". (Hix 1996: 20).

Antes de comenzar a analizar su forma, merece la pena detenerse a reflexionar sobre su orientación, ya que para los invernaderos con esta configuración formal, la orientación puramente sur es la ideal en términos de optimización de la luz solar. ${ }^{12}$ No obstante, si se observa el plano de situación (figura 24), se comprueba que en este caso, el invernadero se encuentra girado hacia el oeste. Cabe preguntarse cuáles son las razones que han llevado a la imposición de esta orientación, por encima de las consideraciones técnicas relativas a la optimización de la luz solar. La respuesta se encuentra en el hecho de que, a pesar de que es un edificio de nueva factura, su emplazamiento, y por tanto su forma $\mathrm{y}$ orientación, venían impuestos previamente como requisitos iniciales del proyecto. Como forma parte del recinto de un jardín histórico del año 1781, y sustituye a los invernaderos anteriores, este invernadero se debía ubicar "adosado al muro de la calle Espalter, lugar donde se encontraban los antiguos invernaderos". (Moleón, 1995: 78). Efectivamente, "el nuevo invernadero de exhibición del Real Jardín Botánico se construyó para sustituir a los que había hecho la Junta para la Ampliación de Estudios en el año de 1929, derribados en 1975. Así pues la ubicación, la planta y la orientación estaban ya preestablecidas" ("Real Jardín Botánico": 1). Es decir, este invernadero está fuertemente condicionado por su emplazamiento.

Recurriendo a una fotografia de la época (figura 25), se observa el gran parecido entre el invernadero de 1993 y el de 1930. Este antiguo invernadero, llamado Estufa del mediodía, se construyó como alternativa al existente Pabellón de Villanueva de 1781 , ya que "la mala orientación de los diseñados por Villanueva, obligó muy pronto a construir uno nuevo, orientado esta vez a mediodía”. (Gomez-Centurión 1999: 71). De la misma manera, la forma alargada del nuevo invernadero está delimitada en su extremo sureste por el Invernadero de Graells o Estufa de las Palmas (figura 26). Ésta se construyó en 1856 , aún está en funcionamiento, y sobre ella se ha publicado que "conserva todo su encanto decimonónico y logra transmitir esa sensación de voluptuoso exotismo". (Gomez-Centurión 1999: 73). Mientras que 
Figura 27. Sección transversal del invernadero de Madrid (AFA Arquitectos, $S L P)$.

Figura 28. Diagrama solar norte-sur del Invernadero Santiago Castroviejo (autora).

Figura 29. Vista del invernadero integrado en el Jardin Botánico (autora).

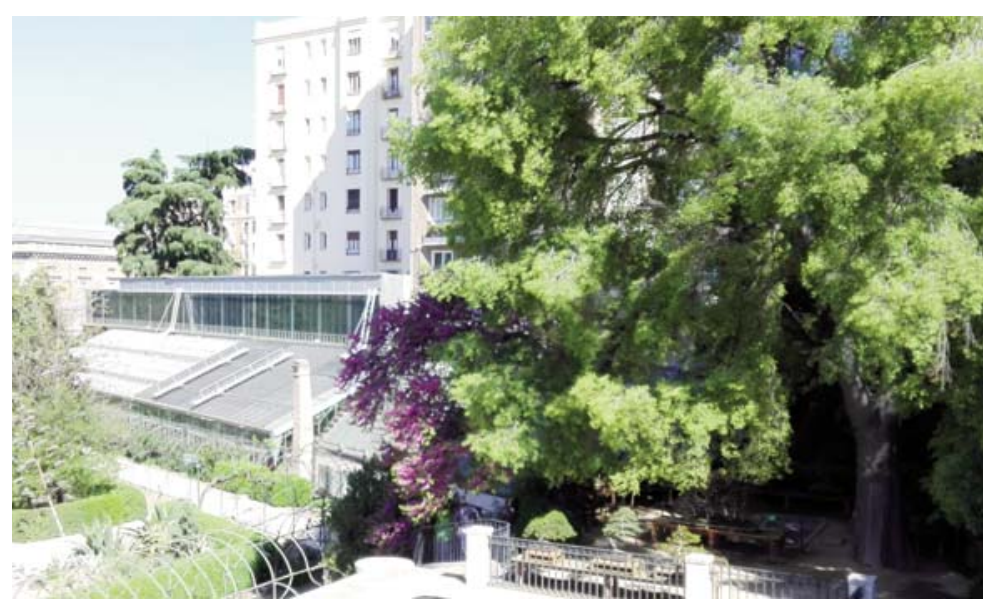

cubierta acristalada está formada por un plano vertical superior ocupado por placas fotovoltaicas, un plano inclinado de vidrio formando $65^{\circ}$ con la vertical y otro vertical de vidrio en su llegada al suelo. De la forma de esta cubierta, compuesta por dos ángulos diferentes, se puede deducir que el objetivo del invernadero no es potenciar la máxima incidencia de la luz solar en un único momento del año, sino que se aproxima más al criterio expuesto por Miller ${ }^{13}$ de "admitir el sol en todas las estaciones, ya que en verano, cuando el sol está alto, el vidrio de cubierta permitirá la radiación en prácticamente todo el espacio, y en invierno, cuando el sol esté bajo, el vidrio frontal dejará pasar los rayos". (Loudon 1817: 8).

Considerando la carta solar de Madrid y el diagrama solar norte-sur (figura 28), se aprecia que los tramos verticales, uno de ellos con placas solares fotovoltaicas, están pensados para captar la luz solar durante los meses de otoño e invierno, así como los rayos horizontales de la mañana durante todo el año. Por otro lado, se observa que el tramo de vidrio inclinado $65^{\circ}$ respecto a la vertical, recibe rayos de luz perpendiculares desde mayo hasta julio. De todo ello se puede concluir que la forma de este invernadero está diseñada para optimizar la luz solar a lo largo de todo el año, especialmente en los meses de primavera y verano. Puesto que los climas que se recrean en su interior - tropical, subtropical y desértico- requieren de una gran cantidad de luz constante, se puede afirmar que la configuración formal de este invernadero coincide con la que sería ideal en cuanto a optimización de la luz solar. Aún así, no se debe olvidar que la orientación más adecuada para este invernadero debería haber sido la puramente sur, de este modo, la cubierta inclinada de vidrio recibiria luz directa a lo largo de toda su longitud, desde mayo hasta agosto, en las horas centrales del día. Sin embargo, al tener una orientación suroeste, la luz directa se recibe en una franja horaria desplazada hacia la tarde. Es decir, la forma óptima en relación a la luz solar se ha adaptado debido a que su emplazamiento y orientación estaban preestablecidos, para de esta manera, lograr una adecuada integración del edificio en su entorno (figura 29).

El invernadero de Madrid se diferencia de los dos invernaderos analizados en lo referente al periodo en que se optimiza la luz solar: el Climatron, con forma cupular simétrica respecto de su centro está diseñado para optimizar la luz solar durante 
Figura 30. Planta superior e inferior del invernadero (AFA Arquitectos, SLP).
Figura 31. El invernadero en construcción, se pueden apreciar los depósitos situados bajo el nivel del suelo (AFA Arquitectos, $S L P)$.

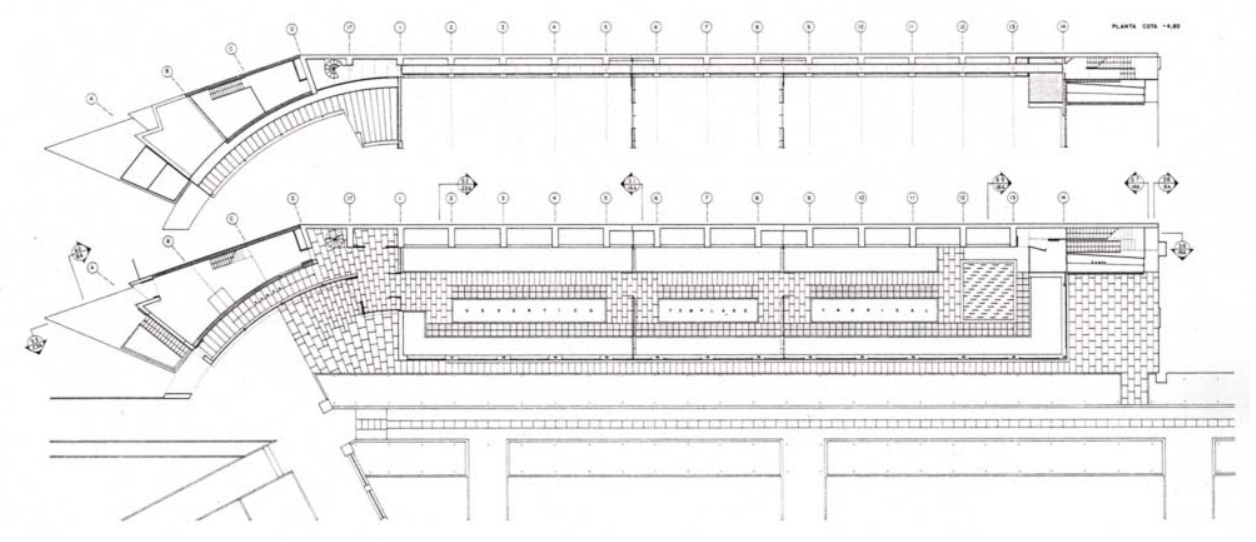

todo el día, todos los días del año. El invernadero con forma de cilindro parabólico de Graz, cuando se dispone en dirección norte-sur, optimiza luz solar a lo largo de toda la trayectoria diaria del sol, tanto por la mañana como por la tarde. Sin embargo, el invernadero de Madrid, capta la máxima luz solar principalmente en primavera y verano.

Esta sección que, tal y como ha quedado demostrado, está fuertemente condicionada por el requisito técnico de optimización de la luz solar, da lugar a un espacio arquitectónico con unas características espaciales concretas. Debido al plano inclinado de su cubierta, en el interior se produce una diferencia de altura entre los lados sur y norte, esta situación espacial permite crear un nuevo modo de recorrido y de relación con el edificio y el jardin: a través de una pasarela superior en el lado

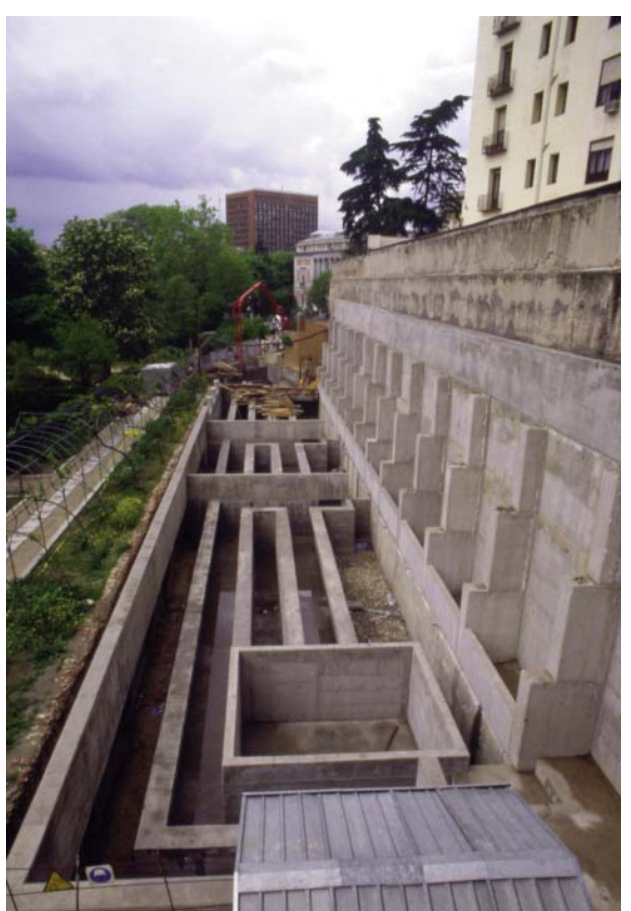

norte, se obtienen vistas de la colección del invernadero y del propio jardín botánico desde un nivel superior (figura 30).

A diferencia de los dos invernaderos anteriores, su sección no está compuesta por una única forma geométrica pura, transparente e inalterada mediante elementos opacos. En este caso, la importancia de mantener el muro de contención, no sólo radica en su valor patrimonial, por formar parte del jardín histórico de 1781, sino también por su papel en el aprovechamiento solar pasivo, ya que protege el invernadero de los vientos del norte, funciona como acumulador de calor y aporta su inercia térmica. El sistema de acondicionamiento térmico tiene una disposición longitudinal, de acuerdo con la forma global del edificio, y funciona del siguiente modo: el aprovechamiento solar activo, se realiza a través de "los paneles solares, situados en la parte superior del invernadero (...) en posición vertical, de tal manera que la radiación solar que interceptan durante el invierno es muy elevada, y muy baja en verano". ("Real Jardín Botáncio": 3). Esta energía se emplea en calentar agua, que se acumula en los depósitos situados bajo el nivel del suelo del invernadero (figura 31), y se "transfiere de manera directa el calor a las plantas por unas rejillas o canales situados en el suelo". (Ref. web 4).

En lo tocante a la exposición, este invernadero "cuenta con una sección de plantas tropicales con alta temperatura y humedad, una sección de plantas de desierto, con muy baja humedad y una sección de plantas templadas en la que debe haber una temperatura y humedad media". (Moleón 1995: 78). Del mismo modo que el invernadero de Graz, la sección del invernadero de Madrid es siempre constante, independientemente de los tres climas diferentes que recrea en su interior, estando delimitados por un plano transversal de 


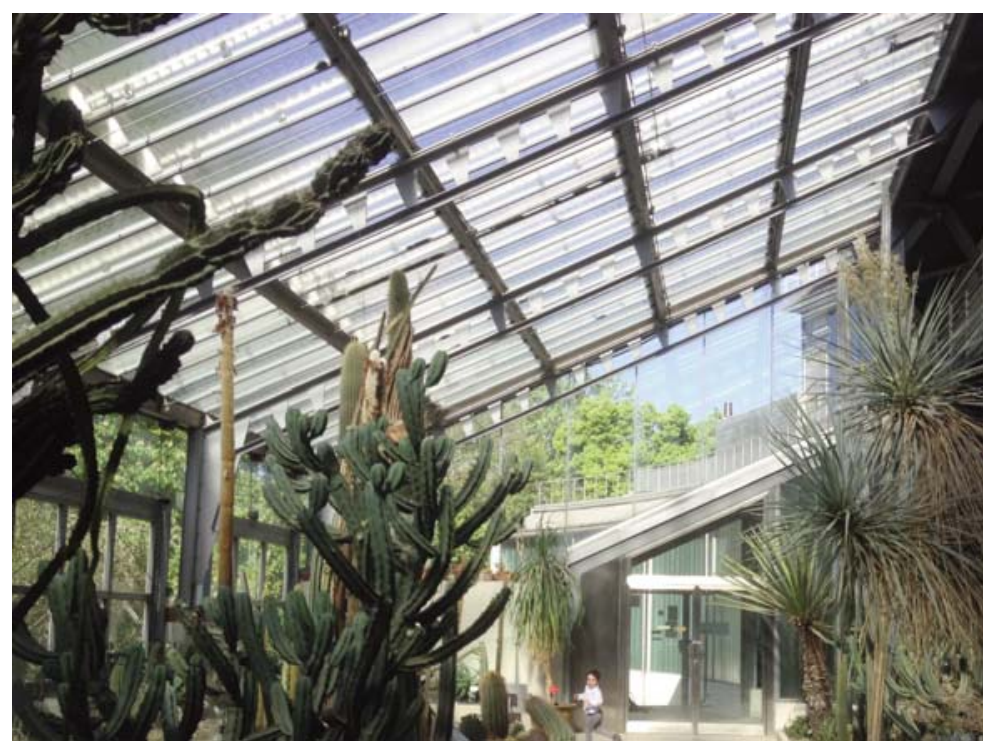

Figura 32. Vista interior del invernadero de Madrid (autora).

vidrio con una puerta que da acceso a cada uno de ellos. La composición longitudinal impuesta para el invernadero, tiene su principal repercusión en las circulaciones, claramente lineales, organizadas en dos calles que se alternan con tres filas de parterres (figura 32). Las rejillas en el suelo son el elemento que regula las variaciones de temperatura y humedad, modulando el efecto por medio de su apertura o cierre en los compartimentos tropical y subtropical, y permanecen cerradas siempre en el desértico. Además, "toda la superficie de cubierta va equipada con un sistema de sombreamiento automático que está concebido para colaborar en la amortiguación de las oscilaciones térmicas interiores y de la excesiva luz solar". ("Invernadero de exhibición” 1995: 50).

La inclusión de este invernadero en los medios de difusión especializados se ha centrado en la óptima respuesta que da a sus diferentes requisitos técnicos, Kenneth Frampton lo describía en Historia critica de la arquitectura moderna como "una nave o hangar sin decoración", y afirmaba que la "adaptabilidad de este volumen" se mantiene "mediante la inclusión de una red homogénea e integrada de instalaciones: energía, luz, calor y ventilación", así como por la necesidad de "articular y expresar tanto la estructura como las instalaciones". (Frampton 2000: 306). Así mismo, Pedro Moleón destacaba en el monográfico sobre Ángel Fernández Alba: su "sistema de control que está concebido para conseguir una mayor eficacia y aprovechamiento energético" (Moleón 1995: 78). En el catálogo de la exposición de la obra del arquitecto del año 2009, Kenneth Frampton describió este invernadero como "una obra decididamente high-tech, no sólo por la precisión de la estructura de acero roblonado que sirve de apoyo a la membrana de cristal, sino también por el sistema de sombreamiento mecánico", y destacaba que "es uno de esos edificios homeostáticos en los que se controla automáticamente la temperatura, la humedad y la radiación solar, para conseguir un eficaz aprovechamiento energético". (AFA Arquitectos Asociados 2008: 78).

Se puede concluir que la forma del invernadero de Madrid está fuertemente condicionada por el requisito inicial del proyecto de integrarse en el jardín histórico al que pertenece. Pero además, su solución formal responde muy positivamente a los requerimientos técnicos de optimización de la luz solar y control climático propios del invernadero. Por todo ello, se puede afirmar que existe un coherente compromiso en la solución conjunta de los factores tanto técnicos como formales del invernadero.

\section{Conclusiones}

Los diagramas solares realizados para cada uno de los invernaderos han permitido realizar una reflexión basada en datos reales, para desentrañar las intenciones de la forma de los invernaderos, en relación al modo en que incide la luz solar en ellos. De esta manera, se han observado cuáles has sido los ajustes que se han hecho a dicha forma para optimizar al máximo la incidencia de luz solar, o por el contrario, cuáles son sus contradicciones a este respecto.

En los tres invernaderos se ha demostrado que, si bien su solución formal responde a requisitos puramente técnicos de optimización de la luz solar, en ellos se da, a la vez, un alto grado de compromiso entre la que sería su forma ideal y factores de otra indole, que intervienen en la configuración del edificio como un todo.

En el caso del Climatron de Misuri, la monumentalidad buscada se consigue mediante la imposición inicial de una forma y un sistema estructural determinados: la cúpula geodésica, que se ha convertido en el principal símbolo del jardín. En los invernaderos de los Jardines Botánicos de la Universidad de Graz, la composición del conjunto, cargada de dinamismo y espacialidad se debe, en gran medida, al empeño por resolver todo el conjunto mediante una única sección parabólica. Mientras que en el Real Jardín Botánico de Madrid, la integración del edificio en el entorno histórico al que pertenece, se consigue mediante la imposición inicial de su emplazamiento, heredado del invernadero anterior al que sustituye. 
Finalmente, para los tres invernaderos ha quedado demostrada la coherente relación y correspondencia que existe entre su forma y los sistemas de acondicionamiento, que permiten cumplir con su función última de recrear un clima diferente al de la latitud en la que se encuentran. En el caso del Climatron, la forma circular coincide con el sistema de tratamiento del aire en el perímetro, que da lugar a un gradiente de temperatura y humedad en el espacio interior, sin necesidad de particiones. En los invernaderos de Graz, los arcos parabólicos estructurales son a la vez los conductos de calefacción. Y en el invernadero de Madrid, la forma longitudinal coincide con el sistema de rejillas que regulan la entrada de calor y humedad en los diferentes compartimentos.

\section{Notas}

1. No se ha localizado ninguna monografia sobre el invernadero del siglo XX, además, en los libros monográficos de historia general del invernadero, el del siglo XX ocupa un capítulo final puntual en la historia del invernadero, mientras que el tema central es el invernadero del siglo XIX. Así mismo, cabe destacar que en los libros de historia de la arquitectura, los invernaderos del siglo XX aparecen como obras particulares, no como una tipología de edificios.

2. En la historia de la construcción de invernaderos del siglo XX en Europa y Estados Unidos, hay un periodo en el que no se construyeron invernaderos, periodo que contiene a la Segunda Guerra Mundial.

En Europa, desde el invernadero Jardin d'hiver en el Jardin des Plantes de París, de 1937, no se construyó ningún otro hasta la Evolution House en Kew Gardens de Londres, de 1952.

En Estados Unidos, desde el invernadero Irwin M. Krohn de Cincinnaty y el United States Botanic Garden Conservatory de Washington D.C, ambos de 1933, no se construyó ningún otro hasta el Climatron del Jardín Botánico de Missouri de 1961.

3. John Claudius Loudon (1783-1843), botánico, químico y agrónomo de formación, centró su práctica profesional en la jardinería y el paisajismo, así como en la experimentación e investigación sobre el invernadero.

4. La colatitud es el ángulo complementario a la latitud, y la declinación, el ángulo formado por el sol y el ecuador.

5. "Un modelo tradicional es la Carta Solar Estereográfica de Fisher-Mattioni que se basa en un sistema de coordenadas angulares donde el radio representa la altura solar, y los ángulos el azimut, que se mide desde el sur $\left(0^{\circ}\right)$ al norte $\left(180^{\circ}\right)$. Sobre este sistema de coordenadas se representan los meses y las horas en función de la altura solar y el azimut para una determinada latitud". (Ref. web 1).

6. La altura (h) es la distancia angular entre el astro $\mathrm{S}$ y el punto $\mathrm{P}$ (punto de intersección entre la vertical que pasa por el astro y el horizonte). (Ref. web 2).

7. Las relaciones geométricas detectadas en el Climatron son semejantes a las que propuso Loudon para la semicúpula de Mackenzie, a la que consideraba "demasiado alta en relación a su longitud", proponiendo como solución "un pequeño segmento de una esfera de mayor radio". (Loudon 1822: 356).

8. Un primer sistema es el de enfriamiento, en dirección oeste-este: el aire entra por el lado oeste y pasa sucesivamente por pulverizadores que aportan humedad, la cual es absorbida por el aire, de modo que éste "está casi saturado de agua y esto reduce la temperatura (...). En su viaje a través del invernadero, el aire gana calor por la radiación solar. Parte de la humedad también es absorbida por las plantas. El resultado (...) es la obtención de los gradientes de temperatura y humedad requeridos en los distintos sectores por los que pasa el aire en su recorrido desde su entrada a su salida por el extremo este". ("Science in overseas" 1961: 362).

9. En la revista Popular Mechanics de julio de 1961, en su artículo "Tropics on the half shell" y en la misma revista en el número de noviembre de 1961, en su artículo "Science in overseas industry".

10. El invernadero de clima frío limita el perimetro noreste, ayudando a crear un frente para el edificio. Aquí la sección parabólica se corta en su lado trasero para crear una fachada más empinada contra la calle (...) La casa tropical, una variación del tema de la sección parabólica se dispone en dirección suroeste.

11. "La ligereza de la estructura y de la envolvente plástica proporcionan una transmisión luminosa del 98\%, record en la historia del invernadero moderno". (Jeska 2008: 152).

12. "Los invernaderos ofrecen un mejor funcionamiento cuando la cubierta transparente se encuentra en un solo lado de su sección transversal, siempre que este lado reciba los rayos solares en un gran ángulo, la mayor parte de la estación de crecimiento de las especies. Esto, por supuesto, está sujeto a limitaciones y variaciones según las circunstancias, y ha dado lugar a una variedad de formas exteriores para los invernaderos y los ángulos de sus cubiertas. Sin embargo, implica la necesidad de situar todos aquellos invernaderos cuya envolvente no sea totalmente transparente, con su fachada acristalada orientada hacia el sur". (Loudon 1822: 354).

13. "En las ediciones séptima y octava del Miller's Dictionary (publicado por última vez en 1768), los artículos Estufa, Sol, Invernadero y algunos otros, están considerablemente ampliados mediante reflexiones y argumentos sobre la pendiente de las cubiertas de vidrio". (Loudon 1817: 8).

\section{Bibliografia}

AAVV. 2007. Botanic Gardens, a living history. Londres: Black Dog Publishing.

AFA ARQuitectos Asociados. Ángel Fernández Alba \& Soledad del Pino Iglesias, fascinación por la tradición moderna. [Cat.] 2008. AFA Madrid: TF Editores.

Alba Fernández, Ángel. 5 Proyectos + 6 Metáforas urbanas. [Cat.] 1991. Madrid: Publicaciones COAM.

ConnaH, Roger (editor) 2001. Volker Giencke Projects. Viena: Springer-Verlag.

BALDASSINI, Niccolò. 1997 enero. "Lo spazio della trasparenza $=$ In the small yet great Graz". L'Arca. $\mathrm{N}^{\circ}$ 111: 20-25.

Blundell-Jones, Peter. 1995 octubre. "Out to Graz". Architectural Review. 200.1196: 71-73.

Chramosta, Walter M. 1995 enero. "Der Zeit ihre Botanil, der Botanik ihre Freiheit". Architektur \& Bauforum. $\mathrm{N}^{\circ} 28174$.

Cunningham, Anne. 2000. Crystal palaces, garden conservatories of the United States. Nueva York: Princeton Architectural Press. 
ELWALL, Robert. 2001 abril. "Climate control". RIBA Journal. Vol. 108, $\mathrm{n}^{\circ}$ 4: 130.

FrAmPTON, Kenneth. 1981 (décima edición 2000). Historia crítica de la arquitectura moderna. Barcelona: Editorial Gustavo Gili, SA.

Freeman, Allen. 1989 marzo. "Reglazing a celebrated dome greenhouse, the Climatron Missouri Botanical Gardens, St. Louis". Journal of the American Institute of Architects. Vol. 78. $\mathrm{N}^{\circ} 3$ 88-89.

Gómez - Centurión, Pilar. 1999. Real Jardín Botánico de Madrid, un jardin ilustrado. Madrid: Lunwerg editores.

GRANT, Fiona. 2013. Glasshouses. Oxford: Shire Publications.

HART, Sara. 2002 mayo. "Buckminster Fuller's dream of spanning great distances are being realized in big projects". Architectural Record.

Hepper, F. Nigel (Editor).1982. Kew: Gradens for science and pleasure. Maryland: Stemmer House Publishers Inc.

HIX, John. 1996, $5^{a}$ edición 2005. The glasshouse. Londres: Phaidon Press LTD.

JESKA, Simone. 2008. Transparent plastics. Stuttgart: Birkhäuser Verlag AG.

KLEIS, Birgitte. 1996 enero. "Vild vaekst - vaeksthuse i den Botaniske Have, Graz, Ostriq". Arketekstur DK. Vol. 40. No 3: A34 - A36.

KoHlmaier, George y von Sartory, Barna. Houses of Glass. A Nineteenth-Century Building Type.1990. (2 $2^{\text {a }}$ ed. 1991). Londres: MIT Press.

KNIGHT, Thomas Andrew. 1808. "Description of a forcing house for grapes; with observations on the best method of constructing houses for other fruits". Transactions of the Horticultural Society of London. Vol. I: 99-102.

KopPELKAMm, Stefan. 1981. Glasshouses and wintergardens of the Nineteenth Century. Nueva York: Rizzoli International Publications.

Leuchars, Robert B. 1854. Practical treatise on the construction, heating and ventilation of hothouses. Boston: John P. Jewett and Company.

Loudon, John Claudius. 1817. Remarks on the construction of hothouses. Londres: printed for J. Taylor at the Architectural Library.

Loudon, John Claudius. 1822. Encyclopedia of gardening. Londres: Longmans, Green. and $\mathrm{CO}$

Miller, Philip. 1754. "Stove" En: Miller's Gardeners Dictionary. Londres: impreso por el autor.

Moleón, Pedro. 1995. Ángel Fernández Alba. Madrid: Fundación Argentaria. TF. Editores.

NeIla GonZÁlez, Francisco Javier. 2004. Arquitectura bioclimática en un entorno sostenible. Madrid. Editorial Munilla-Lería.

Riley, Robert. 1997 julio. "Green chaos. The Climatron and the enclosure of nature". Harvard Design Magazine: 44-46.

Serrano Cermeño, Zoilo. 1992 (3 ${ }^{\mathrm{a}}$ ed. 2005). Cons trucción de invernaderos. Madrid: MundiPrensa Libros, S.A.

WiLKInson, Thomas. 1812. On the best method of constructing a peach-house. Transactions of the Horticultural Society of London. Vol. I.

WOODS, Mary y SWARTZ WARREN, Arete. 1988. Glasshouses, a history of greenhouses, orangeries and conservatories. Londres: Aurum Press Limited.

WrIGHT, William Joseph. 1917. Greenhouses, their construction and equipment. Nueva York: Orange Judd Company.

"Climatron wins Reynolds Award". 1961 abril. Architectural Forum: 5.

"Gewächshäuser der Universität Graz". 1995 enero. Planen Bauen Wohnen. N ${ }^{\circ}$ 155: 24-25.

"Invernadero de exhibición. Real Jardín Botánico. Madrid". 1995. Arquitectura. No 304: 50 - 52.

"Science in overseas Industry". 1961, 9 de noviembre. New Scientist. Vol. 12, $\mathrm{n}^{\circ}$ 260: 362 .

"Tropics on the half shell". 1961 julio. Popular Mechanics. Vol: 116, $\mathrm{n}^{\circ}$ 1: 113-115, 218

"The Climatron: Missouri Botanical Gardens, St.
Louis”. 161 mayo. AIA Journal. Vol. 35. Nº 27 31

"Volker Giencke: glasshouses at the Botanical Gardens, Graz, Austria”. 1996 noviembre. GA Document. N $^{\circ}$ 49: 62-69.

Nota a la bibliografia: cuando no ha sido posible encontrar el autor de un artículo, así como para los catálogos de exposiciones, se ha colocado el título del texto en la posición del nombre del autor, con el fin de facilitar la lectura y la identificación de citas al lector. En las citas de texto, se han tomado las tres primeras palabras del título entre comillas, en la posición del nombre del autor.

\section{Referencias de imágenes}

Ref. web 1: https://fjferrer.webs.ull.es/Apuntes3/Leccion02/31_cartas_solares.html Ref. web 2: http://astroaula.net/recursosdidacticos /actividades/altura-y-azimut/ Ref. web 3: http://www.madehow.com/Volume6/Geodesic-Dome.html

Ref. web 4: http://www.sitiosolar.com Ref. web 5: http://solardat.uoregon.edu/SunChartProgram.html
Fecha final recepción artículos: 30/04/2017

Fecha aceptación:

23/06/2017

Articulo sometido a revisión por dos revisores independientes por el método doble ciego. 